\title{
Chasing the reflected wave back into the heart: a new hypothesis while the jury is still out
}

\author{
This article was published in the following Dove Press journal: \\ Vascular Health and Risk Management \\ 7 June 2011 \\ Number of times this article has been viewed
}

\author{
Ion Codreanu ${ }^{1,2}$ \\ Matthew D Robson ${ }^{2}$ \\ Oliver J Rider ${ }^{2}$ \\ Tammy J Pegg ${ }^{2}$ \\ Bernd A Jung ${ }^{3}$ \\ Constantin A Dasanu ${ }^{4}$ \\ Kieran Clarke' \\ Cameron J Holloway ${ }^{1,2}$ \\ 'Department of Physiology, Anatomy, \\ and Genetics, University of Oxford; \\ ${ }^{2}$ University of Oxford Centre \\ for Clinical Magnetic Resonance \\ Research, Oxford, UK; ${ }^{3}$ Department of \\ Radiology, Medical Physics, University \\ Hospital, Freiburg, Germany; ${ }^{4}$ Saint \\ Francis Hospital and Medical Center, \\ Hartford, CT, USA
}

Background: Arterial stiffness directly influences cardiac function and is independently associated with cardiovascular risk. However, the influence of the aortic reflected pulse pressure wave on left ventricular function has not been well characterized. The aim of this study was to obtain detailed information on regional ventricular wall motion patterns corresponding to the propagation of the reflected aortic wave on ventricular segments.

Methods: Left ventricular wall motion was investigated in a group of healthy volunteers ( $\mathrm{n}=14$, age $23 \pm 3$ years), using cardiac magnetic resonance navigator-gated tissue phase mapping. The left ventricle was divided into 16 segments and regional wall motion was studied in high temporal detail.

Results: Corresponding to the expected timing of the reflected aortic wave reaching the left ventricle, a characteristic "notch" of regional myocardial motion was seen in all radial, circumferential, and longitudinal velocity graphs. This notch was particularly prominent in septal segments adjacent to the left ventricular outflow tract on radial velocity graphs and in anterior and posterior left ventricular segments on circumferential velocity graphs. Similarly, longitudinal velocity graphs demonstrated a brief deceleration in the upward recoil motion of the entire ventricle at the beginning of diastole.

Conclusion: These results provide new insights into the possible influence of the reflected aortic waves on ventricular segments. Although the association with the reflected wave appears to us to be unambiguous, it represents a novel research concept, and further studies enabling the actual recording of the pulse wave are required.

Keywords: arterial pressure wave, reflected wave, ventricular wall motion, cardiovascular magnetic resonance imaging, tissue phase mapping

\section{Introduction}

Left ventricular contraction leads to a pressure wave within the aorta, which is amplified as it travels down the arterial wall in advance of blood flow, and generates an arterial pulse. When the wave reaches major branching points, additional waves are generated and reflected back, which are similarly amplified as they travel back toward the heart. The magnitude and timing of the reflected waves are altered in cardiovascular disease and are modulated by therapies. ${ }^{1}$ Both increased arterial stiffness and increased wave reflections alter cardiac function and are independent predictors of cardiovascular risk. $^{1,2}$ Despite the well-described relationship between arterial pressure waves and cardiac function, ${ }^{3-5}$ the effects of reflected arterial waves on ventricular motion patterns have not been described.

There have been two main schools of thought describing the hemodynamics of the arterial system. The Windkessel theory views the arterial system as a compliance and

\footnotetext{
Correspondence: Ion Codreanu

Department of Physiology,

Anatomy and Genetics,

University of Oxford,

Parks Road, Oxford, OXI 3PT, UK

Tel +441865221869

Fax +44 I865 22I III

Email codrion@yahoo.com
}

submit your manuscript | www.dovepress.com

Dovepress

DOI: $10.2147 /$ VHRM.S20845 
resistance pair, relating pulse pressure to arterial compliance and peripheral resistance. The model assumes that arterial pulse wavelengths are infinite. ${ }^{6}$ The transmission theory views the arterial system as a network of vessels that transmits pulses, which have a certain finite pulse wavelength. The pulse pressure in this model is affected by the magnitude, timing, and sites of pulse wave reflection. ${ }^{6}$ Decreasing heart rate (ie, pulse frequency) by one third increases the pulse wavelength by $50 \%{ }^{6}$ Similarly, increasing vessel radii by $50 \%$ or decreasing arterial compliance by $55.5 \%$ increases the pulse wavelength by $50 \% .^{6}$ These examples demonstrate the complexity of the interactions in the cardiovascular system and the effects of mechanical arterial properties on the pumping ventricle.

The forward travelling (incident) wave depends on the pressure generated by left ventricular contraction and arterial elasticity, while the reflected wave is related to the sites of wave reflection and mechanical arterial properties. ${ }^{7-9}$ The reflected wave alters arterial wall motion with an amplitude almost similar to that of the incident wave, giving the pulse wave in large arteries a dicrotic shape (Figure 1). It has been reported that changes in pulse wave contour could readily be explained on the basis of increased stiffness of the aorta and early return of wave reflection. ${ }^{10,11}$

However, how this reflected pulse wave alters left ventricular motion, and whether it contributes to diastolic function in

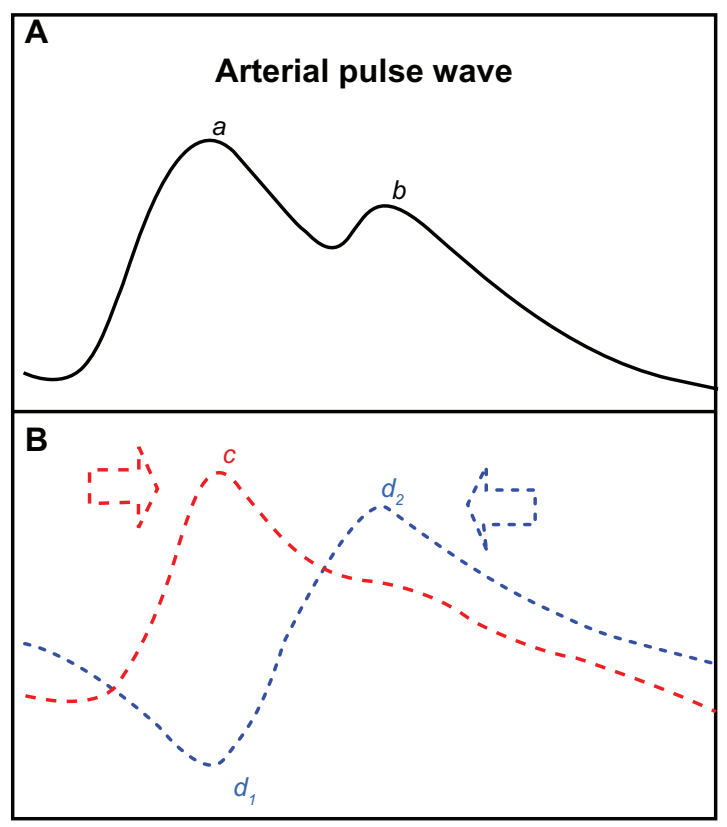

Figure I Schematic representation of the pulse pressure wave (A) and its components (B). The main peak (a) caused by the incident wave (c) and the subsequent notch $(b)$ caused by the outward directed part of the reflected wave $\left(d_{2}\right)$ give the pulse pressure wave a dicrotic shape. healthy and diseased hearts, is not established. To determine the effects of the reflected aortic wave on the heart, we used a cardiac magnetic resonance technique with high temporal resolution (navigator-gated tissue phase mapping) to record changes in three-dimensional left ventricular wall motion in normal healthy subjects.

\section{Methods}

Cardiac magnetic resonance examinations were performed using a 1.5 Tesla Siemens Sonata scanner (Siemans Medical Solutions USA, Inc, Malvern, PA) on 14 healthy male volunteers. At the initial assessment visit, a medical history was taken and a screening examination was performed to exclude any cardiovascular, endocrine, or neuromuscular disease. An electrocardiogram was performed for an accurate assessment of heart rate. Baseline characteristics of the study participants are provided in Table 1.

Cine images for navigator-gated high temporal resolution tissue phase mapping were acquired using a black blood segmented k-space gradient echo sequence ${ }^{12,13}$ (repetition time $=13.8 \mathrm{msec}$, echo time $=5.0 \mathrm{msec}$, flip angle $=15^{\circ}$, bandwidth $=650 \mathrm{~Hz} /$ pixel, field of view $=400 \times 300 \mathrm{~mm}$, matrix $=256 \times 96$ ) with a temporal resolution of $13.8 \mathrm{msec}$. Three short axis images were acquired for the left ventricular base, mid ventricle, and apex. Tissue phase mapping analysis was performed using customized software (Matlab, version 6.5, Mathworks, Natick, MA). The endocardial and epicardial borders were manually contoured for the base, mid ventricle, and apex in each phase of the cardiac cycle. For tissue phase mapping analysis, the left ventricle was divided into 16 segments (six basal, six middle, and four apical) according to the American Society of Echocardiography and American Heart Association models. ${ }^{14}$ Inplane velocities were transformed into an internal polar coordinate system positioned at the center of mass of the left ventricle. As a result, motion parameters are described in terms of radial, circumferential, and longitudinal velocities. Global and

Table I Baseline characteristics of study participants

\begin{tabular}{lr}
\hline Characteristic & Mean \pm SD \\
\hline Age (years) & $22.8 \pm 2.9$ \\
Height $(\mathrm{cm})$ & $186.1 \pm 8.1$ \\
Weight $(\mathrm{kg})$ & $82.2 \pm 8.3$ \\
Body mass index & $23.8 \pm 2.0$ \\
Heart rate (beats/min) & $56.6 \pm 7.1$ \\
Systolic blood pressure $(\mathrm{mmHg})$ & $119.1 \pm 7.8$ \\
Diastolic blood pressure $(\mathrm{mmHg})$ & $75.1 \pm 4.3$ \\
Left ventricular ejection fraction $(\%)$ & $67.7 \pm 5.9$ \\
\hline
\end{tabular}

Abbreviation: SD, standard deviation. 
regional velocity time courses were calculated as the mean of all myocardial pixels within the segmentation mask or within the 16 segments, respectively. All myocardial velocities are expressed as $\mathrm{cm} / \mathrm{sec}$. Circumferential velocities are described as clockwise or counterclockwise, as viewed from the ventricular apex. The duration of the cardiac cycle was determined by the R-R interval on the electrocardiogram, with end systole defined as the smallest left ventricular cavity, as previously described..$^{15}$ The study was conducted according to the principles of the Declaration of Helsinki and was approved by a local Oxfordshire Clinical Research Ethics Committee. Each subject provided written informed consent.

\section{Results}

The radial, circumferential, and longitudinal motion patterns of the heart are demonstrated in Figures 2-4. In addition to the large motion patterns observed in each segment, a wave of altered motion (Figures 2-4), is consistently seen in early diastole, corresponding to the expected timing of the reflected aortic wave reaching the left ventricle (Figure 1).

\section{Effects on ventricular radial motion}

Radial velocities from individual left ventricular segments, as well as average values for the left ventricular base, mid ventricle, and apex during a cardiac cycle, are shown in Figure 2. Positive values demonstrate myocardial wall motion toward the center of the ventricle, while negative values reflect an outward motion.
The radial notch in early diastole has an upright deflection and resembles the dicrotic notch of the arterial pulse wave (Figure 2) and corresponds to a brief thrust of inward movement in early diastole (when the notch is above zero) or a sudden deceleration in the radial expansion of the ventricular wall (when the notch is below zero). On the segmental velocity curves, this early diastolic notch was particularly prominent in the septal segments $(2,3,8$, and 9). In the lateral segments (5-6 at the ventricular base, $11-12$ at the mid ventricular level) the notch was above zero, indicating an additional thrust of inward movement at the beginning of diastole. In most other segments, the notch was below zero, indicating a brief deceleration in radial expansion. This early diastolic notch was more prominent in the basal segments and less prominent in the apical segments, which are located further away from the attachment of the aorta to the left ventricular outflow tract (ie, segments 12-16).

\section{Effects on ventricular longitudinal motion}

The velocity of the longitudinal left ventricular segments during a cardiac cycle is shown in Figure 3. These graphs represent average values for all volunteers. Positive values reflect downward motion along the longitudinal left ventricular axis (ie, toward the apex) and negative values show upward motion (ie, toward the base). The early diastolic notch observed in all left ventricular segments (Figure 3) demonstrates a brief deceleration in the upward recoil motion of the entire ventricle at the beginning of diastole,

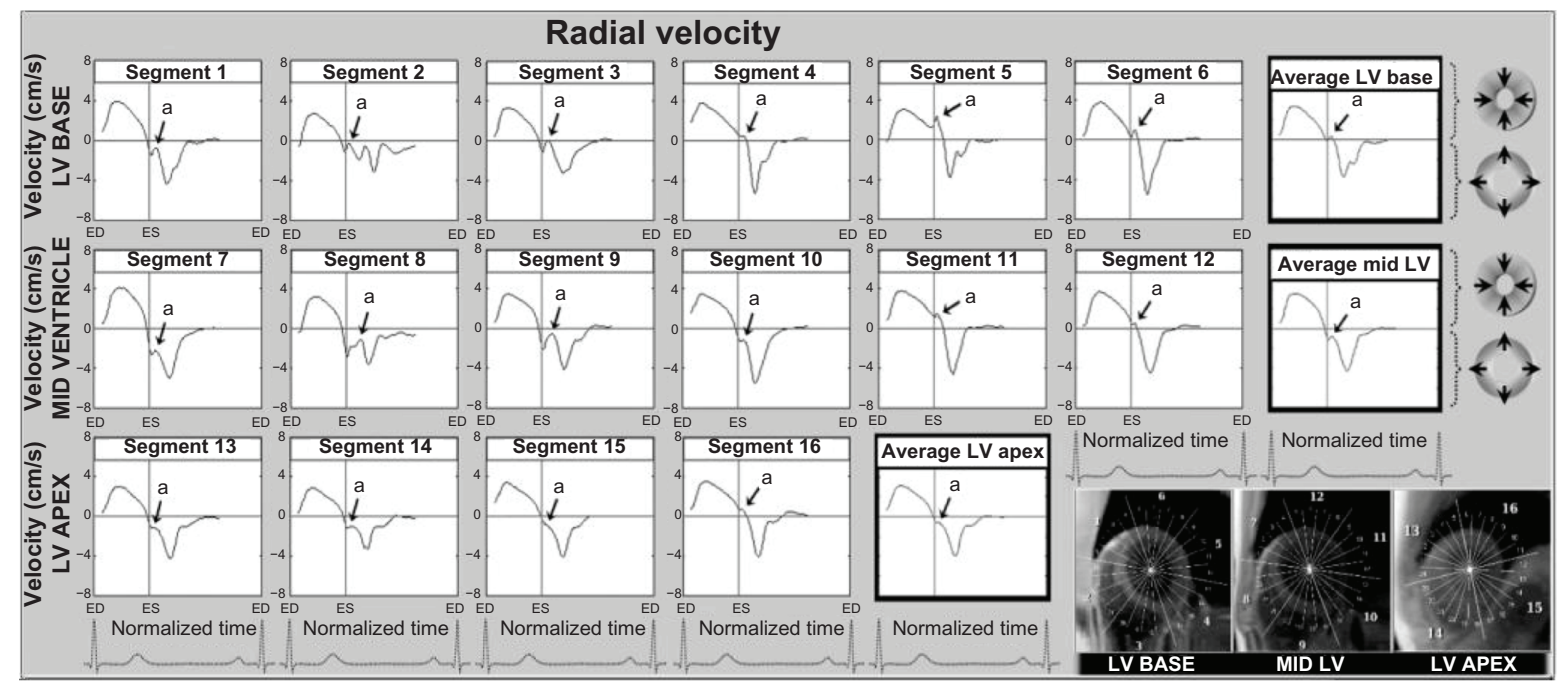

Figure 2 Radial velocity graphs for individual left ventricular segments (American Heart Association segmentation model) during a cardiac cycle, showing the influence of reflected aortic pressure waves on radial motion in early diastole. The graphs represent average myocardial velocities for all volunteers. Positive values show inward motion toward the center of the ventricle, while negative values show outward expansion. Average velocities for basal, mid, and apical slices are shown in bold outline. The arrows (a) point toward an upward directed notch in early diastole, synonymous with the notch seen on the aortic pulse wave (Figure I). The right lower images show left ventricular short axis images of the base, mid ventricle and apex, divided into 16 segments, which corresponds to the individual left ventricular segments. Abbreviations: ED, end diastole; ES, end systole; LV, left ventricle. 


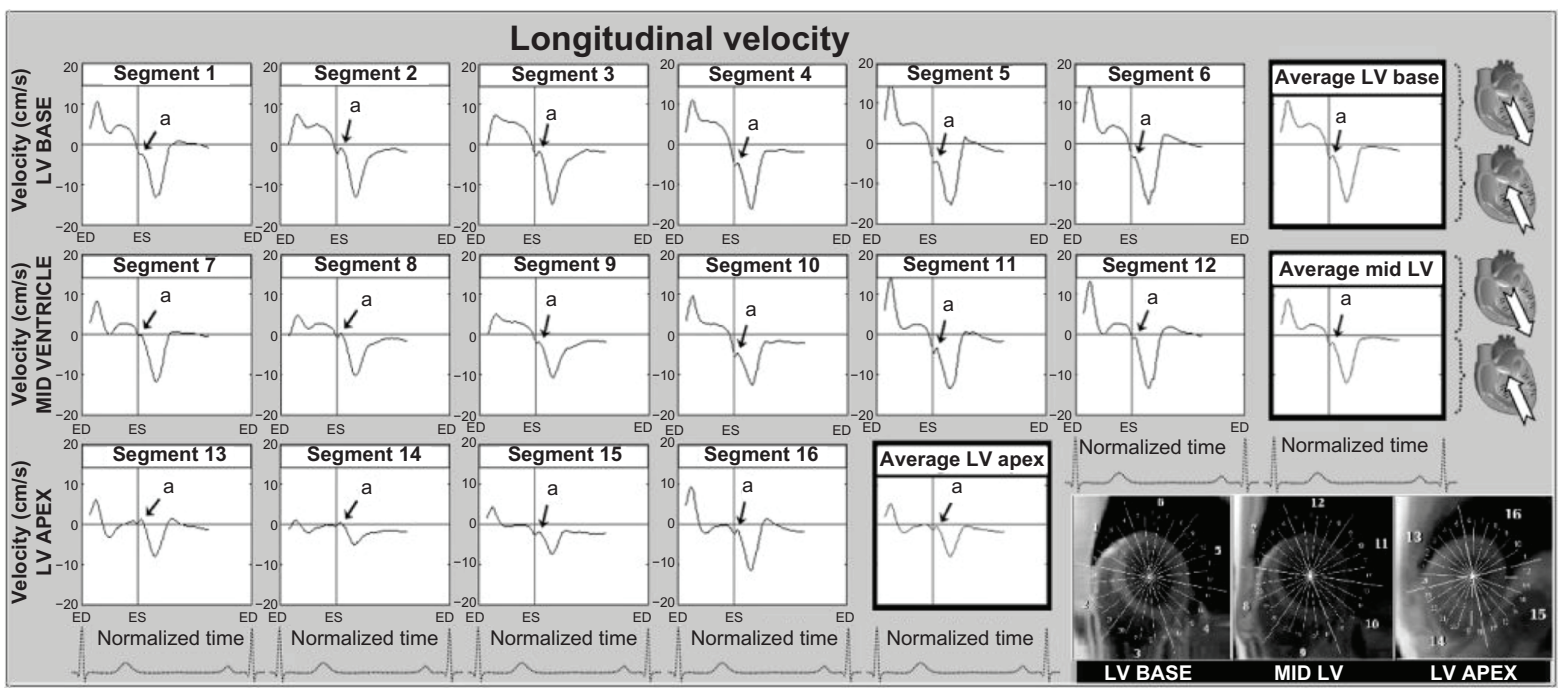

Figure 3 Longitudinal velocity graphs for individual left ventricular segments (American Heart Association segmentation model) showing the influence of reflected aortic pressure waves on longitudinal motion in early diastole. The graphs represent average values for all volunteers. Positive values correspond to downward motion along the ventricular longitudinal axis (ie, toward the apex), while negative values reflect upward displacement (ie, toward the base). Average velocities for basal, mid, and apical slices are shown in bold outline. The arrows (a) show a brief notch in early diastole corresponding to the expected impact with the reflected wave travelling in an opposite direction. The right lower images show left ventricular short axis images of the base, mid ventricle, and apex, divided into 16 segments, which correspond to the individual left ventricular segments.

Abbreviations: ED, end diastole; ES, end systole; LV, left ventricle.

corresponding to the expected impact with the reflected pulse wave travelling in the opposite direction.

\section{Effects on ventricular circumferential motion}

The circumferential or rotational velocities of individual left ventricular segments, as well as average values for the left ventricular base, mid ventricle, and apex are shown in Figure 4. Positive values reflect clockwise rotation and negative values reflect counterclockwise rotation, as viewed from the apex. A brief clockwise motion occurred at the beginning of diastole in most basal segments (Figure 4). At the left ventricular apex and mid ventricle, the notch was superimposed on a larger wave of clockwise rotation of the left ventricular apex during ventricular untwisting (Figure 4).

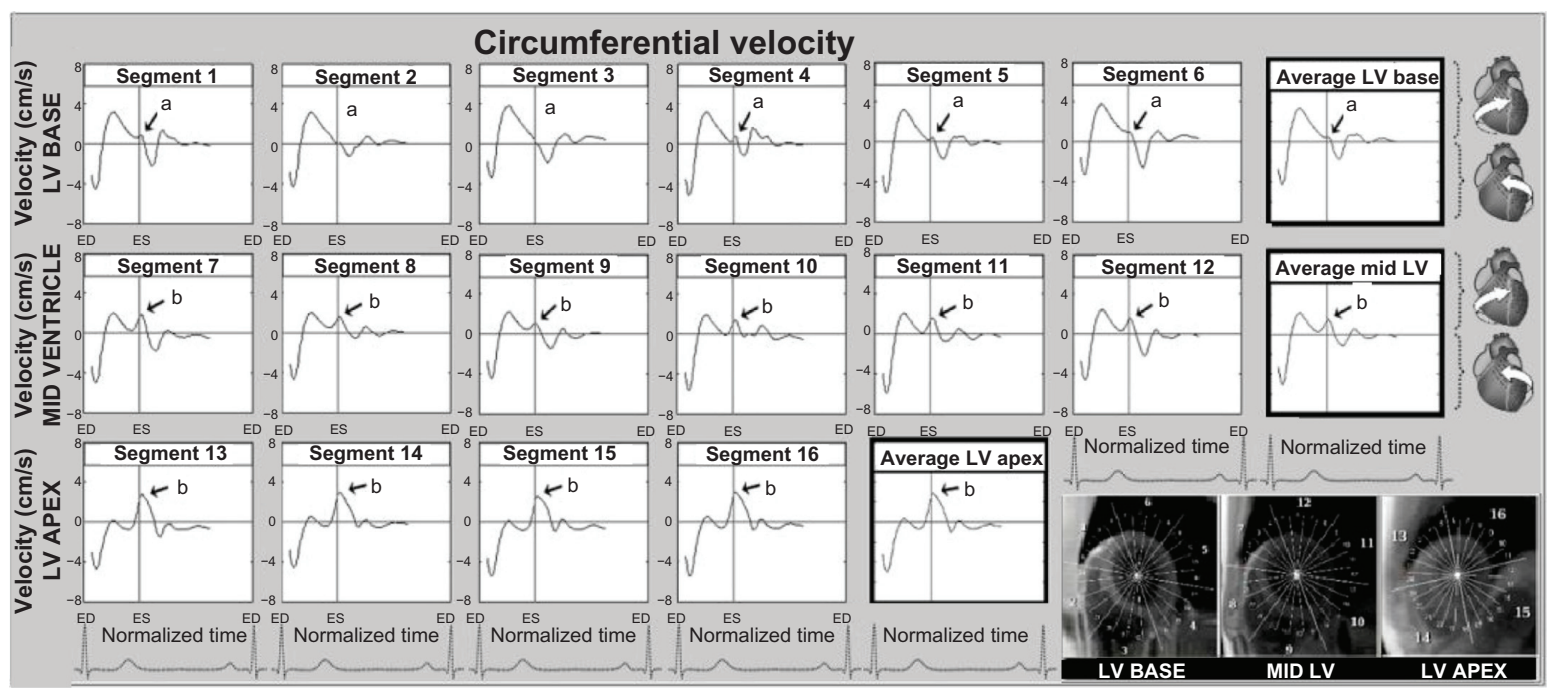

Figure 4 Circumferential velocity graphs for individual left ventricular segments (American Heart Association segmentation model) showing the influence of reflected aortic pressure waves on circumferential motion in early diastole. The graphs represent average values for all volunteers. Positive values correspond to clockwise rotation of the left ventricle as viewed from the apex, while negative values reflect counterclockwise motion. Average velocities for basal, mid, and apical slices are shown in bold outline. The arrows (a) show a brief notch of clockwise rotation in early diastole. At the ventricular apex and mid ventricle (segments 7-16), the notch was superimposed on a larger wave of clockwise rotation of the ventricular apex (recoil untwisting) after repolarization (b). The right lower images show left ventricular short axis images of the base, mid ventricle, and apex, divided into 16 segments, which corresponds to the individual left ventricular segments.

Abbreviations: ED, end diastole; ES, end systole; LV, left ventricle. 


\section{Discussion}

The arterial pressure wave represents a superposition of two separate waves, that is, the incident wave travelling from the heart toward the periphery, and the reflected wave travelling from the periphery and the sites of wave reflection toward the heart. ${ }^{7-9}$ As opposed to blood flow, the pulse pressure wave spreads almost instantaneously and is amplified as it travels. The reflected aortic wave travels at a similar speed to the incident wave but in an opposite direction and is amplified as it travels toward the heart, reaching it in late systole or early diastole. Moreover, the reflected wave alters arterial wall motion with an amplitude almost similar to that of the incident wave, suggesting it influences the motion patterns of the aorta and left ventricle.

For the first time, we have linked early diastolic segmental motion patterns and the associated changes in radial, circumferential, and longitudinal left ventricular motion with the reflected aortic pulse wave reaching the left ventricle. The upright notch on the radial velocity graphs in early diastole (Figure 2) corresponds to a sudden deceleration in the commencement of radial relaxation, or even a brief inward motion in separate segments. As the front of the reflected wave travelling toward the heart was directed inwards (Figure 1), it is conceivable that it caused a brief inward displacement, or a brief deceleration in the ongoing radial expansion when reaching the left ventricle (Figure 2). The subsequent outward directed part of the reflected wave (Figure 1), responsible for the dicrotic shape of the pulse wave (Figure 1), was superimposed on the ongoing radial expansion in diastole. The prominence of this early diastolic notch in septal segments $(2,3,8$, and 9) may be explained by the close proximity of the aorta to the septum, the inline wave propagation on these adjacent segments having a greater mechanical effect (Figure 5). This is further supported by a similar shape of the reflected wave in the aorta and membranous septum, which form a continuous structure with no mechanical barriers for pulse wave propagation.

Longitudinal motion patterns reveal a sudden deceleration in the upward motion of the entire ventricle at the beginning of diastole (Figure 3), which may be explained by the impact of the reflected pressure wave travelling in an opposite direction. Moreover, the brief early diastolic notch of clockwise rotation seen on circumferential velocity graphs (Figure 4) suggests the reflected wave leads to a brief clockwise rotation when reaching these segments. The orientation of the aortic arch and attachment of the ascending aorta to the left ventricle

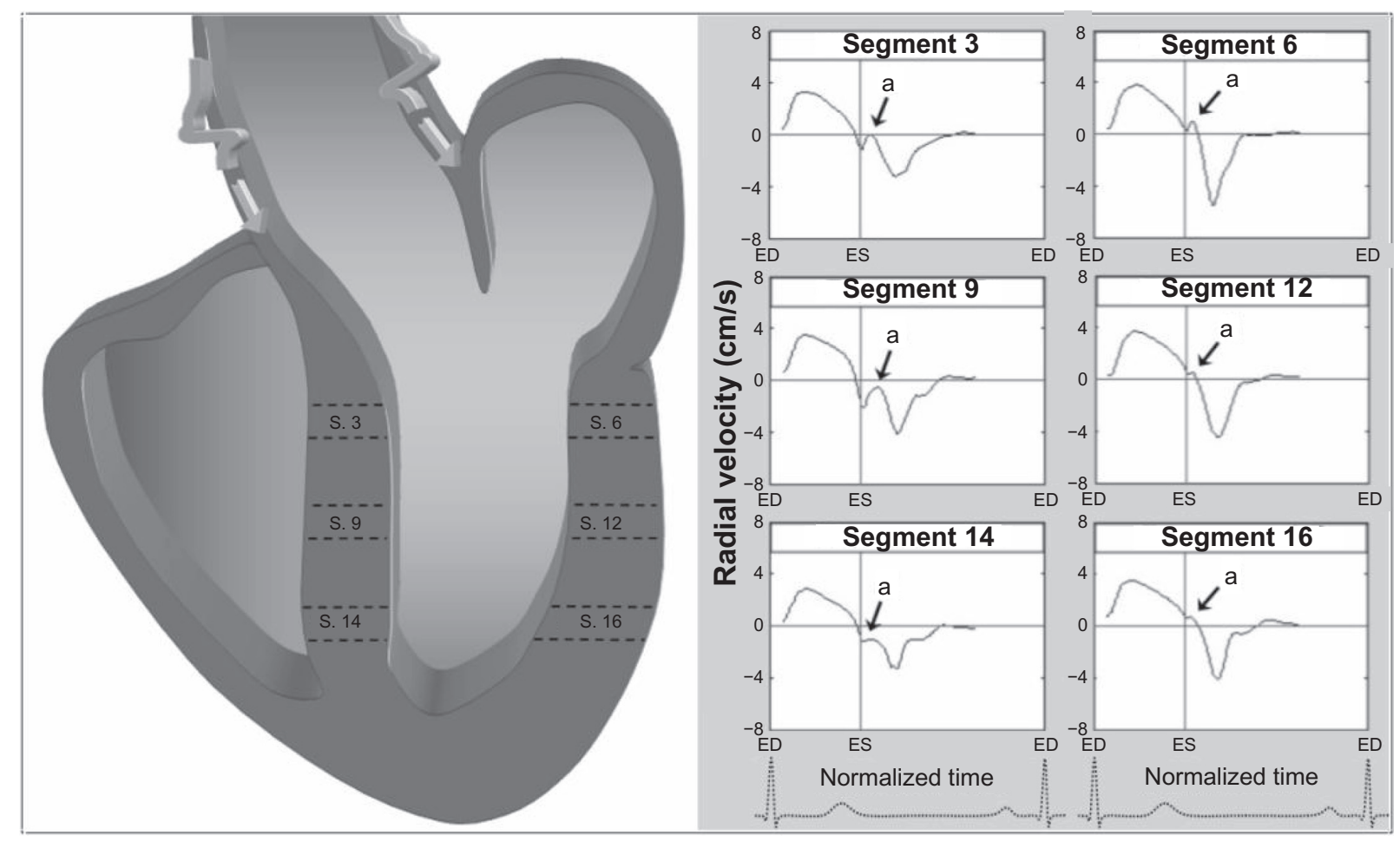

Figure $\mathbf{5}$ Schematic representation of the effect of reflected wave propagation on ventricular segments, demonstrating a greater influence of reflected aortic pressures waves on septal segments which are in continuity with the aorta, compared with lateral segments which are not in direct continuity. The arrows (a) shows an upright notch in early diastole corresponding to propagation of the reflected wave on left ventricular segments. The graphs represent average values for all volunteers. Positive values show inward motion toward the center of the ventricle, while negative values show outward expansion.

Abbreviations: ED, end diastole; ES, end systole; LV, left ventricle. 
may explain this mechanically driven clockwise motion. A schematic representation of this motion is provided in Figure 6.

The early diastolic notch that we have described has been reported in studies of radial left ventricular motion using other techniques, with various proposed explanations. ${ }^{16-18}$ The notch is also observed in the first studies of myocardial motion obtained using high temporal resolution magnetic resonance tissue phase mapping. ${ }^{12}$ Garcia et al suggested that the notch reflects geometric changes caused by asynchronous contraction and ventricular interdependence. ${ }^{16}$ Sengupta et al suggested the notch was associated with asynchronous deformation of subendocardial and subepicardial left ventricular wall layers. ${ }^{18}$ In line with these hypotheses, the reflected wave may influence the epicardium, mid-myocardium, and endocardium to different degrees. The magnitude and force of the reflected pulse waves are sufficiently high to cause an aortic pulsation (Figure 1), and the effect on ventricular segments is consistent with the recorded asynchronous deformation of subendocardial and subepicardial layers, ${ }^{18}$ with a sudden interruption of late systolic lengthening ${ }^{17}$ and with accentuation in stiff arteries and ischemic heart disease. ${ }^{19}$

There is no expected difference between the timing of the reflected wave reaching the left ventricle and the commencement of aortic valve closure, which is consistent with the experiments by Remme et al. ${ }^{17}$ Given that the pulse wave spreads almost instantaneously throughout the body, propagation of the reflected wave is expected to be synchronous throughout the left ventricle, as previously shown. ${ }^{17}$ Myocardial motion secondary to the reflected aortic wave would be expected to decrease after a sudden drop in systemic pressure, as Remme et al observed after mitral valve stenting (the amplitude of both incident and reflected waves would be lower in this case). ${ }^{17}$ In addition, placing a stent in the aortic valve orifice and inflating it to the point of preventing aortic valve closure led to a mechanical obstacle, preventing propagation of the reflected wave from the aorta to the ventricular segments. ${ }^{17}$ However, replacing the valve did not obstruct the wave propagation, if the size of the prosthesis matched the size of the aortic lumen. ${ }^{17}$

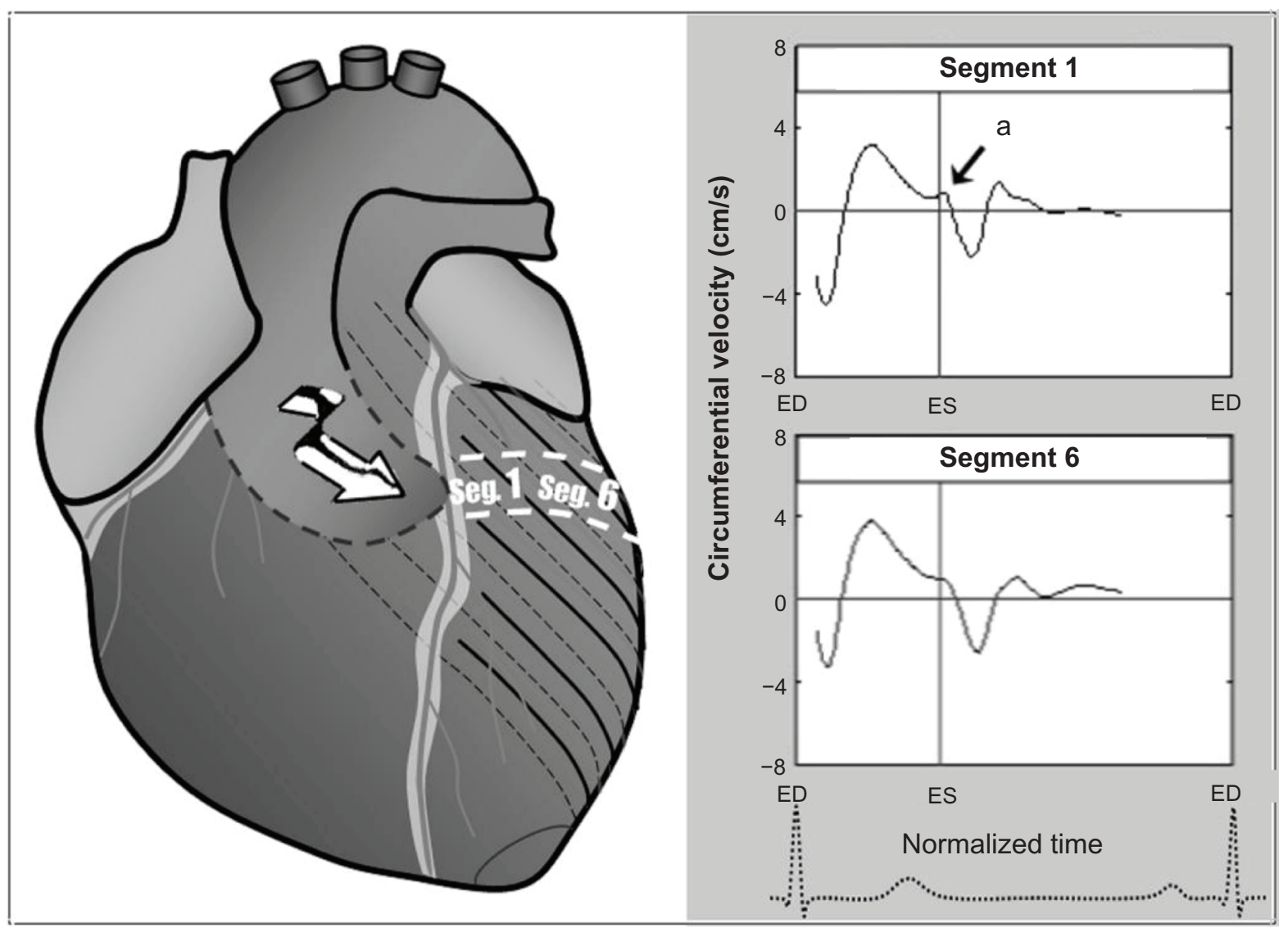

Figure 6 The brief notch of clockwise rotation (a) in the anterior ventricular segments could be explained by potential propagation of the reflected wave on these segments, given the orientation of the aortic arch. The graphs represent average values for all volunteers. Positive values show clockwise rotation, while negative values reflect counterclockwise motion.

Abbreviations: ED, end diastole; ES, end systole; Seg, segment. 
Although the effects of aortic reflected waves on ventricular motion pattern in disease states have not been studied, it has been established that early return of reflected wave boosts (central) systolic blood pressure, increasing the load on the heart and boosting the amplitude of the pressure pulse. ${ }^{20}$ Increased peripheral wave reflection has actually been recorded in chronic heart failure. ${ }^{3}$ Therefore, it is entirely plausible that the reflected pulse wave may adversely influence ventricular function in disease states. Pulse wave velocity is a strong predictor for cardiovascular and all-cause mortality in hypertension ${ }^{21}$ and chronic renal disease, ${ }^{22-25}$ and is independently related to coronary outcome in atherosclerosis, ${ }^{26}$ suggesting that it plays both a functional and prognostic role in cardiovascular disease. In addition, the aortic wave reflections may also have important implications for optimization of therapy. ${ }^{27}$ For example, from all the therapeutic agents used in essential hypertension, arterial wave reflections decreased only following perindopril, outlining a potential role for this drug to alter the effect of the reflected wave on cardiovascular function. ${ }^{28}$

Our results suggest that propagation of the reflected pulse wave may affect early diastolic radial, circumferential, and longitudinal ventricular motion in healthy subjects. In addition, we have previously described the shape of this late systolic/early diastolic notch change in an older age group, affecting the pattern of ventricular motion in early diastole. ${ }^{29}$ We have previously described that the pattern of diastolic motion is different in patients with ischemic heart disease. ${ }^{29}$ The myocardial velocity graphs for two such patients are provided in Figure 7. Large artery stiffening may be both a cause and a consequence of atherosclerosis, and there is clear experimental and clinical evidence that large artery stiffening promotes myocardial ischemia secondary to central pulse pressure elevation. ${ }^{26} \mathrm{~A}$ combination of excessive inward movement during the phase of isovolumic relaxation accompanied by reduced peak systolic and diastolic wall velocities

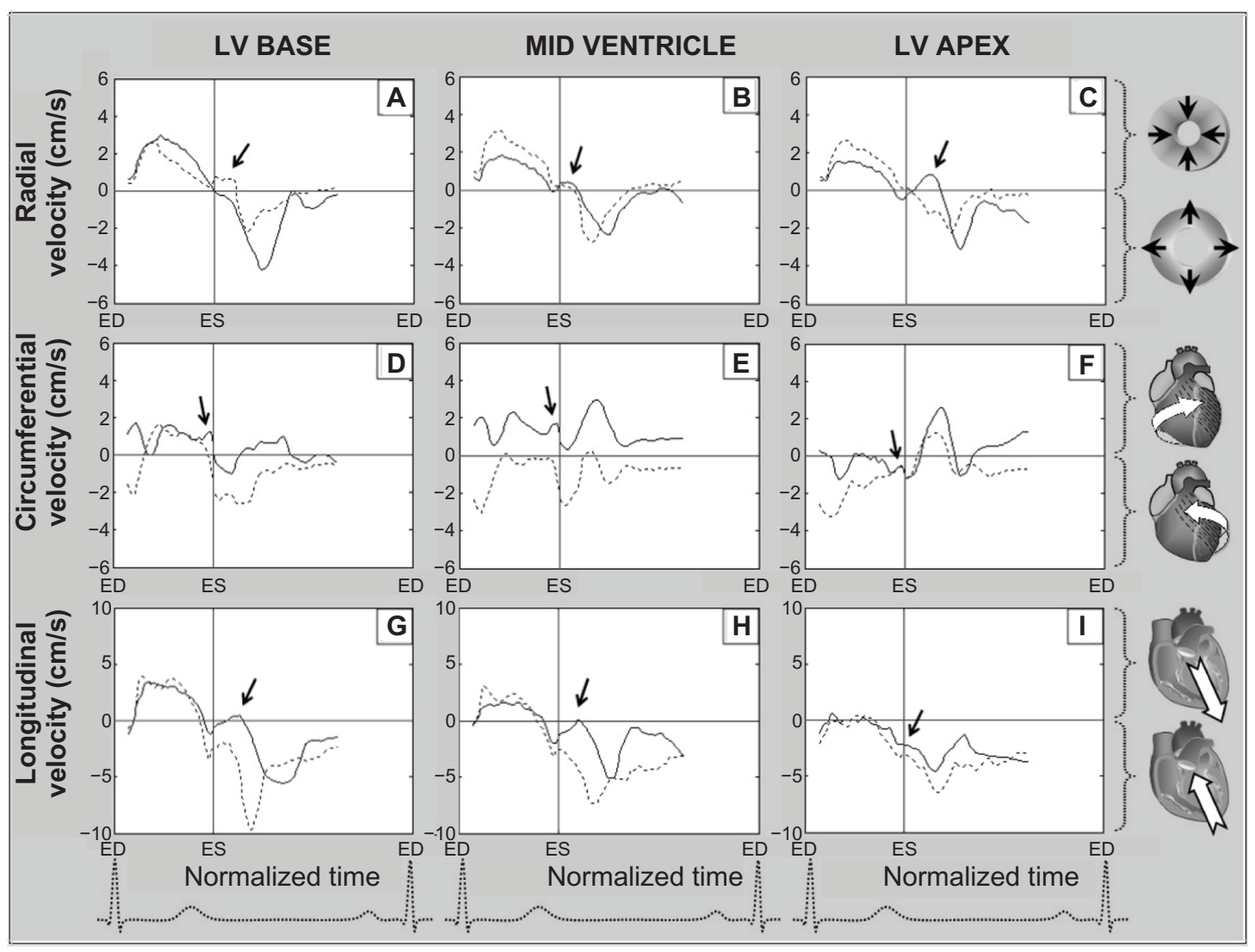

Figure 7 Radial, circumferential, and longitudinal velocity graphs obtained in a patient with a left ventricular scar (solid line) and in a subject with ischemic heart disease and associated peripheral vascular disease (dotted line). The ventricular base represents average values for segments I-6, the mid ventricle represents average values for segments 7-12, and the apex represents average values for segments 13-16 (American Heart Association segmentation model). Positive radial velocities show inward motion and negative values show outward motion. Positive circumferential velocities show clockwise rotation and negative values show counterclockwise rotation. Positive longitudinal velocities show downward motion toward the apex, while negative values show upward motion along the ventricular longitudinal axis. Of note is the paradoxical amplification of the early diastolic notch on radial velocity graphs from the ventricular base toward the apex (arrows pointing toward the solid line graph on $\mathrm{A}=>\mathrm{B}=>\mathrm{C}$ ), likely representing amplification of reflected wave on scar tissue.

Abbreviations: ED, end diastole; ES, end systole; LV, left ventricle. 
in patients with coronary artery disease ${ }^{16,19}$ is also consistent with systemic arterial involvement and accentuated wave reflections in most patients with stenotic coronary arteries. The findings are similar to those recorded in our 69-year-old patient with ischemic heart disease and associated peripheral vascular disease (Figure 7). In the patient with a massive left ventricular scar, it seems the reflected wave freely propagated along the scar tissue without being absorbed. Moreover, the wave was apparently amplified as it travelled toward the apex, like in a rigid tube (arrows in Figures 7A, 7B, and 7C). The findings can serve as a distinct example of the effects of reflected waves on ventricular motion patterns and their clinical relevance. However, these represent individual cases, and further studies involving larger groups of patients with different cardiac and vascular pathology are required to validate our data. Furthermore, quantifying the reflected wave may provide important information in disease states. Methods which enable reflected wave quantification are important considerations. ${ }^{30}$ Consequently, the effects of reflected waves on ventricular motion patterns in cardiovascular disease require further investigation and may yield new insights into the mechanisms of diastolic dysfunction in cardiac pathology.

The high temporal resolution navigator-gated tissue phase mapping technique has allowed registration of tiny details of three-dimensional motion of myocardial segments, but the registration of pulse wave propagation on the aortic wall was not possible in the magnetic resonance scanner. We are describing this concept for the first time and believe it deserves further attention, at least as a possible cause for the recorded motion patterns.

\section{Acknowledgments}

This research was funded by the British Heart Foundation, and was supported by the Oxford Partnership Comprehensive Biomedical Research Centre, with funding from the Department of Health's National Institute for Health Research Biomedical Research Centre.

\section{Disclosure}

The authors report no conflicts of interest in this work.

\section{References}

1. Palombo C, Malshi E, Morizzo C, et al. Arterial wave reflection during antihypertensive therapy with barnidipine: a 6-month, openlabel study using an integrated cardiovascular ultrasound approach in patients with newly diagnosed hypertension. Clin Ther. 2009;31(12): 2873-2885

2. Safar ME, Blacher J, Pannier B, et al. Central pulse pressure and mortality in end-stage renal disease. Hypertension. 2002;39(3):735-738.
3. Curtis SL, Zambanini A, Mayet J, et al. Reduced systolic wave generation and increased peripheral wave reflection in chronic heart failure. Am J Physiol Heart Circ Physiol. 2007;293(1):H557-H562.

4. Jones CJ, Sugawara M. "Wavefronts" in the aorta - implications for the mechanisms of left ventricular ejection and aortic valve closure. Cardiovasc Res. 1993;27(11):1902-1905.

5. Laskey WK, Kussmaul WG. Arterial wave reflection in heart failure. Circulation. 1987;75(4):711-722.

6. Mohiuddin MW, Laine GA, Quick CM. Increase in pulse wavelength causes the systemic arterial tree to degenerate into a classical windkessel. Am J Physiol Heart Circ Physiol. 2007;293(2):H1164-H1171.

7. Berger DS, Robinson KA, Shroff SG. Wave propagation in coupled left ventricle-arterial system: implications for aortic pressure. Hypertension. 1996;27(5):1079-1089.

8. Khir AW, O’Brien A, Gibbs JS, Parker KH. Determination of wave speed and wave separation in the arteries. J Biomech. 2001;34(9): 1145-1155.

9. Pythoud F, Stergiopulos N, Meister JJ. Separation of arterial pressure waves into their forward and backward running components. J Biomech Eng. 1996;118(3):295-301.

10. O'Rourke MF, Gallagher DE. Pulse wave analysis. J Hypertens Suppl. 1996;14(5):S147-S157.

11. O'Rourke MF, Kelly RP. Wave reflection in the systemic circulation and its implications in ventricular function. J Hypertens. 1993;11(4): 327-337.

12. Jung B, Foll D, Bottler P, Petersen S, Hennig J, Markl M. Detailed analysis of myocardial motion in volunteers and patients using hightemporal-resolution MR tissue phase mapping. J Magn Reson Imaging. 2006;24(5):1033-1039.

13. Jung B, Mark1 M, Foll D, Hennig J. Investigating myocardial motion by MRI using tissue phase mapping. Eur J Cardiothorac Surg. 2006;29 Suppl 1:S150-S157.

14. Cerqueira MD, Weissman NJ, Dilsizian V, et al. Standardized myocardial segmentation and nomenclature for tomographic imaging of the heart: a statement for healthcare professionals from the Cardiac Imaging Committee of the Council on Clinical Cardiology of the American Heart Association. Circulation. 2002;105(4):539-542.

15. Codreanu I, Robson MD, Golding SJ, Jung BA, Clarke K, Holloway CJ. Longitudinally and circumferentially directed movements of the left ventricle studied by cardiovascular magnetic resonance phase contrast velocity mapping. J Cardiovasc Magn Reson. 2010; 12(48):48.

16. Garcia MJ, Rodriguez L, Ares M, et al. Myocardial wall velocity assessment by pulsed Doppler tissue imaging: characteristic findings in normal subjects. Am Heart J. 1996;132(3):648-656.

17. Remme EW, Lyseggen E, Helle-Valle T, et al. Mechanisms of preejection and postejection velocity spikes in left ventricular myocardium: Interaction between wall deformation and valve events. Circulation. 2008;118(4):373-380.

18. Sengupta PP, Khandheria BK, Korinek J, Wang J, Belohlavek M. Biphasic tissue Doppler waveforms during isovolumic phases are associated with asynchronous deformation of subendocardial and subepicardial layers. J Appl Physiol. 2005;99(3):1104-1111.

19. Gibson DG, Prewitt TA, Brown DJ. Analysis of left ventricular wall movement during isovolumic relaxation and its relation to coronary artery disease. Br Heart J. 1976;38(10):1010-1019.

20. Kips JG, Rietzschel ER, De Buyzere ML, et al. Evaluation of noninvasive methods to assess wave reflection and pulse transit time from the pressure waveform alone. Hypertension. 2009;53(2):142-149.

21. Laurent S, Boutouyrie P, Asmar R, et al. Aortic stiffness is an independent predictor of all-cause and cardiovascular mortality in hypertensive patients. Hypertension. 2001;37(5):1236-1241.

22. Blacher J, Guerin AP, Pannier B, Marchais SJ, Safar ME, London GM. Impact of aortic stiffness on survival in end-stage renal disease. Circulation. 1999;99(18):2434-2439.

23. Blacher J, Safar ME, Pannier B, Guerin AP, Marchais SJ, London GM. Prognostic significance of arterial stiffness measurements in end-stage renal disease patients. Curr Opin Nephrol Hypertens. 2002;11(6): 629-634. 
24. Guerin AP, Blacher J, Pannier B, Marchais SJ, Safar ME, London GM. Impact of aortic stiffness attenuation on survival of patients in end-stage renal failure. Circulation. 2001;103(7):987-992.

25. London GM, Blacher J, Pannier B, Guerin AP, Marchais SJ, Safar ME. Arterial wave reflections and survival in end-stage renal failure. Hypertension. 2001;38(3):434-438.

26. Kingwell BA, Ahimastos AA. Arterial stiffness and coronary ischaemic disease. Adv Cardiol. 2007;44:125-138

27. De Luca N, Mallion JM, O'Rourke MF, et al. Regression of left ventricular mass in hypertensive patients treated with perindopril/ indapamide as a first-line combination: the REASON echocardiography study. Am J Hypertens. 2004;17(8):660-667.
28. Pannier BM, Guerin AP, Marchais SJ, London GM. Different aortic reflection wave responses following long-term angiotensin-converting enzyme inhibition and beta-blocker in essential hypertension. Clin Exp Pharmacol Physiol. 2001;28(12):1074-1077.

29. Codreanu I, Holloway CJ, Pegg TJ, Robson MD, Neubauer S, Clarke K. New details of reflected pressure wave propagation on left ventricular segments. J Cardiovasc Magn Reson. 2010;12 Suppl 1:127.

30. Westerhof BE, Guelen I, Westerhof N, Karemaker JM, Avolio A. Quantification of wave reflection in the human aorta from pressure alone: A proof of principle. Hypertension. 2006;48(4):5 95-601.

\section{Publish your work in this journal}

Vascular Health and Risk Management is an international, peerreviewed journal of therapeutics and risk management, focusing on concise rapid reporting of clinical studies on the processes involved in the maintenance of vascular health; the monitoring, prevention and treatment of vascular disease and its sequelae; and the involvement of metabolic disorders, particularly diabetes. This journal is indexed on PubMed Central and MedLine. The manuscript management system is completely online and includes a very quick and fair peer-review system, which is all easy to use. Visit http://www.dovepress.com/ testimonials.php to read real quotes from published authors.

Submit your manuscript here: http://www.dovepress.com/vascular-health-and-risk-management-journal 